\title{
Erratum
}

\section{Erratum: Lakunina et al., "Somatostatin-Expressing Interneurons in the Auditory Cortex Mediate Sustained Suppression by Spectral Surround"}

In the article "Somatostatin-Expressing Interneurons in the Auditory Cortex Mediate Sustained Suppression by Spectral Surround," by Anna A. Lakunina, Matthew B. Nardoci, Yashar Ahmadian, and Santiago Jaramillo, which appeared on pages 3564-3575 of the April 29, 2020 issue, the authors would like to address the following error. Panel $\boldsymbol{G}$ in Figure 1 included a brain slice that was not intended to be associated with Figure 1.

The legend for panel $\boldsymbol{G}$ of Figure 1 should read as follows: $\boldsymbol{G}$, Coronal brain slice (AP: $-2.78 \mathrm{~mm}$ ) showing different electrode tracks: magenta (DiI) and blue (DiD). Recordings were performed at multiple sites along each penetration. AUDp, Primary auditory area; AUDd, dorsal auditory area, according to Allen Mouse Brain Atlas.

This correction does not affect the interpretation or conclusions of the article. The correct Figure 1 appears below. The online version has been corrected.

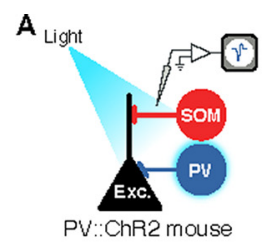

$\mathbf{D}_{\text {Lig }}$

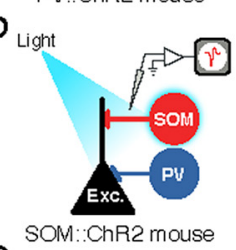

G

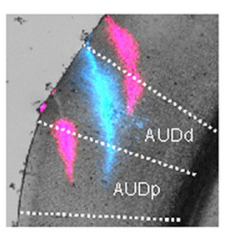

B

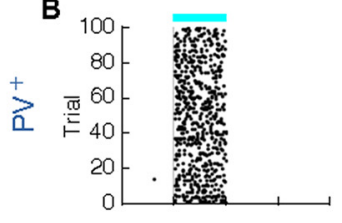

E
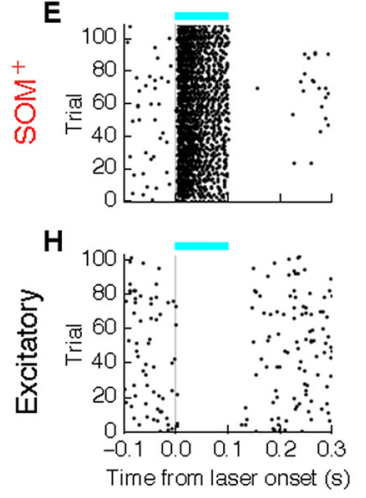

C

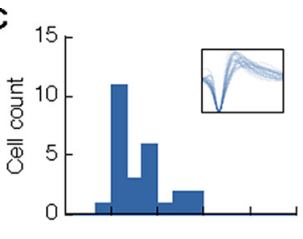

F
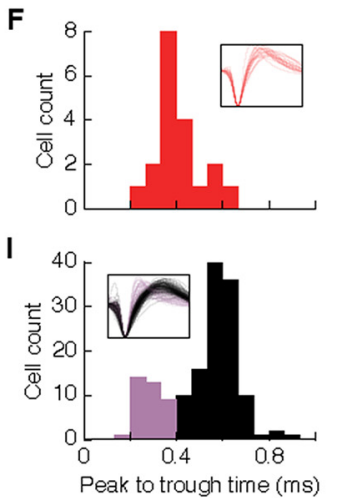

J

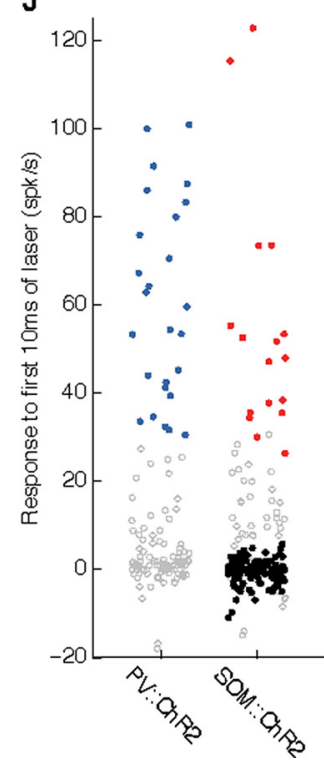

Figure 1. 